\title{
Leg Load Increase Training Using Soft Gait-assistive Suit and Its Experimental Validation
}

\author{
Tetsuro Miyazaki, ${ }^{1 *}$ Toshihiro Kawase, ${ }^{1,2}$ Takahiro Kanno, ${ }^{1}$ and Kenji Kawashima ${ }^{1}$ \\ ${ }^{1}$ Institute of Biomaterials and Bioengineering, Tokyo Medical and Dental University, \\ 2-3-10 Kanda Surugadai, Chiyoda-ku, Tokyo 101-0062, Japan \\ ${ }^{2}$ Institute of Innovative Research, Tokyo Institute of Technology, \\ 4259 Nagatsuta-cho, Midori-ku, Yokohama, Kanagawa 226-8503, Japan
}

(Received September 26, 2019; accepted January 24, 2020)

Keywords: leg load increase training, soft gait-assistive suit, pneumatic artificial rubber muscle, gait phase detection, pressure-based control

A gait training method that can adjust the target load for a trainee is required to meet various demands for training. In this paper, a leg load increase training method using a soft gait-assistive suit is proposed. The soft gait-assistive suit is a power assistive device that helps a trainee walk by actuating a pneumatic artificial rubber muscle (PARM) attached to the trainee's leg. In the proposed leg load increase training, an external force acting as a load is applied to the trainee's leg using the suit so that the muscle activity increases throughout the gait cycle. We focused on the trainee's joint torque trajectories during walking, and the PARMs are actuated to increase them. The effectiveness of the proposed training method was verified by gait experiments on two trainees using a treadmill. The leg electromyogram (EMG) of the trainee wearing the gait-assistive suit was measured, and its change caused by the applied load was observed. From the experimental results, we confirmed that the proposed training method increased the load of the hip extension and flexion of the two trainees, and the load of ankle dorsiflexion of one trainee.

\section{Introduction}

\subsection{Background}

One of the major causes of elderly people needing primary nursing care is motor system disorders $^{(1,2)}$ and an appropriate exercise habit is important for realizing a healthy and highlongevity society. For example, conventional research studies have shown that resistance training can improve muscle performance even in the elderly. ${ }^{(3-6)}$ Owing to the fitness boom in recent years, the training demand for a wide range of age groups is increasing.

Gait training using a treadmill is generally performed widely in a training gym. In basic gait training, trainees walk with their own weight. When a trainee is a senior person whose *Corresponding author: e-mail: tmiyazaki.bmc@tmd.ac.jp https://doi.org/10.18494/SAM.2020.2633 
purpose of the training is to improve physical fitness, the load of its own weight may be too large in some training stages. In this case, conventional gait training methods with the trainee's weight compensation will be effective. For example, there are methods using lifting force with a harness, ${ }^{(7)}$ water buoyancy force in a pool, ${ }^{(8)}$ pneumatic pressure force in a balloon, ${ }^{(9)}$ and so forth. On the other hand, some trainees may require a harder training condition to improve their motor skills. For example, an electric treadmill SKILLRUN ${ }^{(10)}$ developed by Technogym can improve the kicking force of the leg by controlling the rotational resistance of the running belt. As an example of rehabilitation, Kim and Hwang studied the effect of gait training on a sand field for patients with chronic stroke, and found that the sand gait training can improve their gait endurance more effectively than the gait training on firm ground. ${ }^{(11)}$ There are many gait training methods; however, these methods intend either to assist or increase the load of the trainees. To meet the various demands for training, a gait training method that can adjust the target load appropriately for each trainee is required, and a training system that can both reduce and increase the trainee's load will be suitable for the purpose.

As one of the conventional research studies, the authors developed a gait-assistive suit that reduces leg load during walking. ${ }^{(12)}$ This gait-assistive suit helps the trainees walk by actuating a pneumatic artificial rubber muscle (PARM) attached to the leg. The PARMs contract at appropriate timings in gait cycles, as determined by a proposed gait phase detection method. In addition, the gait-assistive suit has no electrical sensors in the attachment unit that the trainee wears; therefore, the suit can also be used in an underwater environment. ${ }^{(13)}$ By using the gait-assistive suit, the gait training that can adjust the appropriate load in each trainee will be realized. There are two ways of the load tuning using the gait-assistive suit, namely, reducing and increasing the load. As described in Ref. 12, the load reduction experiment using the gaitassistive suit was carried out, and its performance was confirmed. However, the load increase experiment using the gait-assistive suit has not been evaluated.

\subsection{Contributions of this paper}

In this paper, a leg load increase training method using a gait-assistive suit is proposed. A detailed analysis of human gait motion was carried out, ${ }^{(14)}$ and the gait data obtained were used for controlling the suit. In the load reduction experiment described in Ref. 12, the assisting force was applied during the activity period of the nearby muscle where the PARM was attached, and the electromyogram (EMG) signal was reduced in the gait cycle. On the other hand, in the proposed load increase training, an external force acting as a load is applied to the trainee's leg using the suit so that the muscle activity increases throughout the gait cycle. Concretely, we focused on the trainee's joint torque trajectories during walking, and the PARMs are actuated to increase them. When time-series data in each joint torque of the trainee are given, the PARM contracts and generates a counter-torque that varies along with the trainee's gait phase. By using the proposed training method, the load of the leg muscle during walking will increase. The effectiveness of the proposed training method is verified by gait experiments on a treadmill. The leg EMG of the trainee wearing the gait-assistive suit is measured, and its change caused by the applied load is observed. 
This paper is organized as follows. In Sect. 2, we describe a mechanism and a pneumatic control system of the gait-assistive suit. In Sect. 3, we describe a control algorithm of the gaitassistive suit for the leg load increase training. In Sect. 4, we discuss the experiments conducted to confirm the effectiveness of the proposed training method. Concluding remarks are given in Sect. 5 .

\section{Gait-assistive Suit}

\subsection{Mechanism of gait-assistive suit}

The gait-assistive suit ${ }^{(12)}$ used in this paper is shown in Fig. 1, which was upgraded from the suit described in Ref. 12. A shoulder belt was installed to hang a waist supporter and prevent the deviation of the suit supporters' position caused by the PARM contraction force. Guide rails were added to knee supporters to constrain the side slip of the thigh front PARM. The gait-assistive suit has a mechanism that fixes the PARM on the trainee's leg, and motions of the thigh, calf, and foot are assisted by the shrinking force of the PARM. The PARMs are attached onto target muscles, which include the vastus lateralis of the front thigh, semitendinosus of the back thigh, gastrocnemius of the back calf, and tibialis anterior of the front calf. Eight PARMs in total are used on the left and right legs. In Fig. 1, $\mathrm{R}$ and $\mathrm{L}$ mean the right leg and the left leg, TF and TB mean the thigh front and the thigh back, and CF and CB mean the calf front and the calf back, respectively. The gait-assistive suit consists of only lightweight and soft components as the PARMs, a shoulder belt, a waist supporter, knee supporters, shoes, and nylon belts. The total weight of the attachment unit is about $1 \mathrm{~kg}$. During the trainee's walking, each PARM is actuated in proper timing and the leg motion is assisted. The proper assist timing is detected by a method using pressure information in the PARMs described in Sect. 3.1. The gaitassistive suit has a unique feature that the attachment unit has no electrical components such as

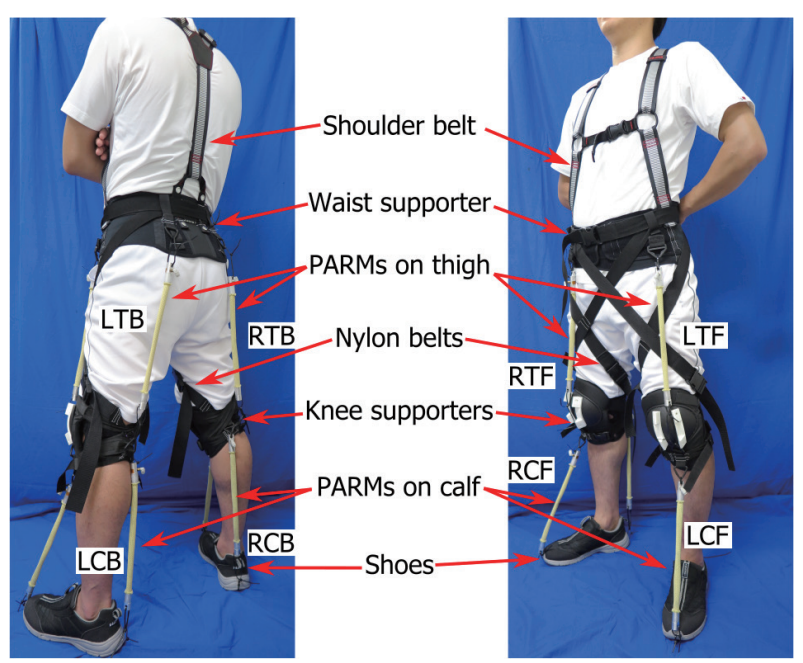

Fig. 1. (Color online) Gait-assistive suit (Left: back; Right: front). 
sensors. The pressure in the PARM is measured by the pressure sensor in the control unit that is connected to the attachment unit only by air tubes. The control unit is described in Sect. 2.2. Owing to this feature, the trainee can wear and use the suit easily without any time-consuming sensor calibration procedure. The mechanism of the attachment unit is designed assuming that the trainee is a regular Japanese male of about $170 \mathrm{~cm}$ height and about $65 \mathrm{~kg}$ weight. The PARM length is $250 \mathrm{~mm}$, and its stroke is $75 \mathrm{~mm}$. The maximum contraction force when the pressure of $400 \mathrm{kPa}$ is supplied is about $300 \mathrm{~N}$. This contraction force gives $15 \mathrm{Nm}$ torque on the joint when the length between the body surface and the joint center is assumed to be $50 \mathrm{~mm}$. This torque is the same as $10 \%$ of the maximum leg joint torque of the target trainee mentioned above during walking. ${ }^{(14)}$

\subsection{Pneumatic control system}

The pneumatic control system ${ }^{(12)}$ of the gait-assistive suit is shown in Fig. 2. The suit shown in Fig. 1 is connected to the control unit by the air tubes. The control unit consists of a computer, $\mathrm{A} / \mathrm{D}$ and $\mathrm{D} / \mathrm{A}$ boards, a compressor, an air tank, pressure regulators, servo valves (FESTO, MPYE5-1/4-010-B), two-port hand valves, and pressure sensors (SMC Corp., PSE510). During one gait cycle, the CB PARM placed on the gastrocnemius muscle is stretched together with the muscle during the preswing phase, which is a transition period from the stance phase to the swing phase, and contracts rapidly after the toe kicks the ground. This unique motion is used for the gait phase detection in the PARM control. Concretely, the CB PARM is initially pressurized and then its airflow is shutout by closing the two-port hand valves. The CB PARM becomes a pressurized closed chamber similar to a balloon. It deforms its shape along with the cyclic gait motion, and its inner pressure also changes. The large pressure change will occur during the preswing phase and is used for the gait phase detection. Gait cycle time is calculated from the detected timing in the preswing phase. In a conventional study, ${ }^{(14)}$ the activity period of each muscle and the trajectory of the joint torque in one gait cycle were analyzed in detail.

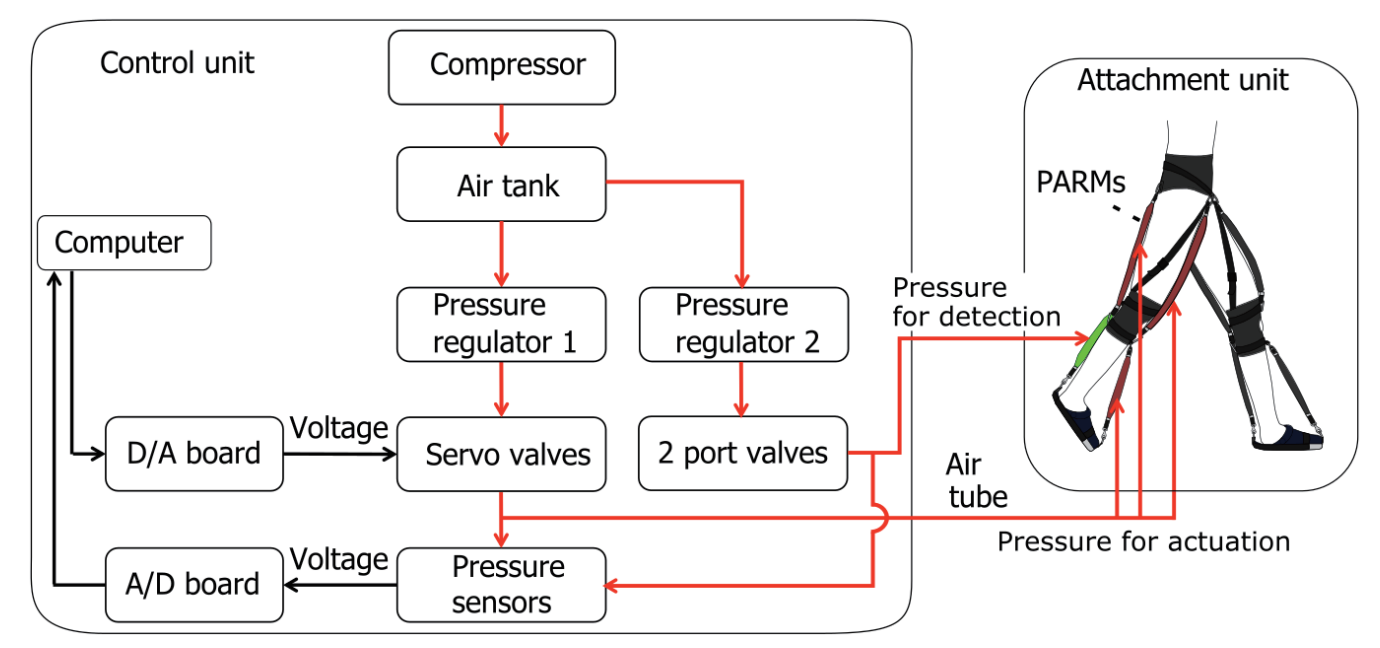

Fig. 2. (Color online) Pneumatic control system. 
We use the results described in Ref. 14 for designing the target pressure of the control system to actuate the PARM. The PARM other than CB is driven by the servo valve and controlled by the pressure feedback controller, as shown in Fig. 3. In Fig. $3, P(\mathrm{kPa})$ is pressure, $P_{\text {ref }}(\mathrm{kPa})$ is target pressure, $u(\mathrm{~V})$ is control voltage, $K_{p}(\mathrm{~V} / \mathrm{kPa})$ is proportional gain, $K_{i}(\mathrm{~V} / \mathrm{kPa}$ s) is integral gain, and $s$ is the Laplace operator.

\section{Control Algorithm of Gait-assistive Suit}

\subsection{Gait phase detection method}

Here, the gait phase detection method, ${ }^{(12)}$ that is, the detection of the preswing phase timing from the pressure trajectory of the CB PARM, is described. For example, when the trainee walks wearing the pressurized RCB PARM, the experimental data samples of pressure and the pressure derivative in the RCB PARM are as shown in Fig. 4. The pressure data obtained at a sampling frequency of $500 \mathrm{~Hz}$ is measured by the control system, and its noise is removed using

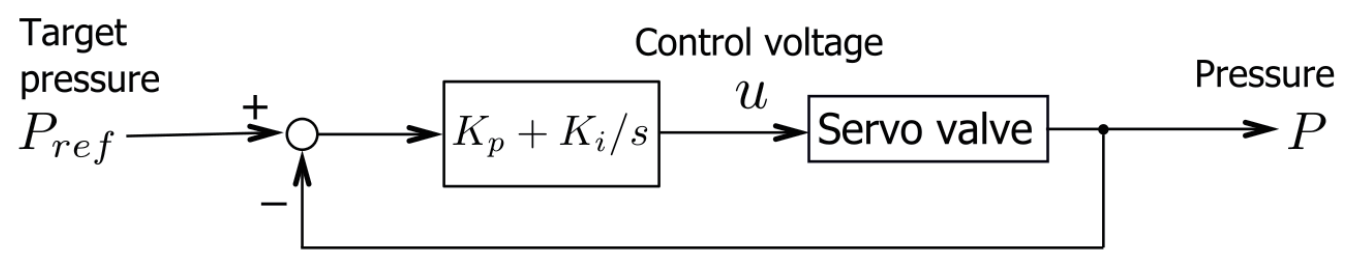

Fig. 3. Block diagram of pressure feedback controller.

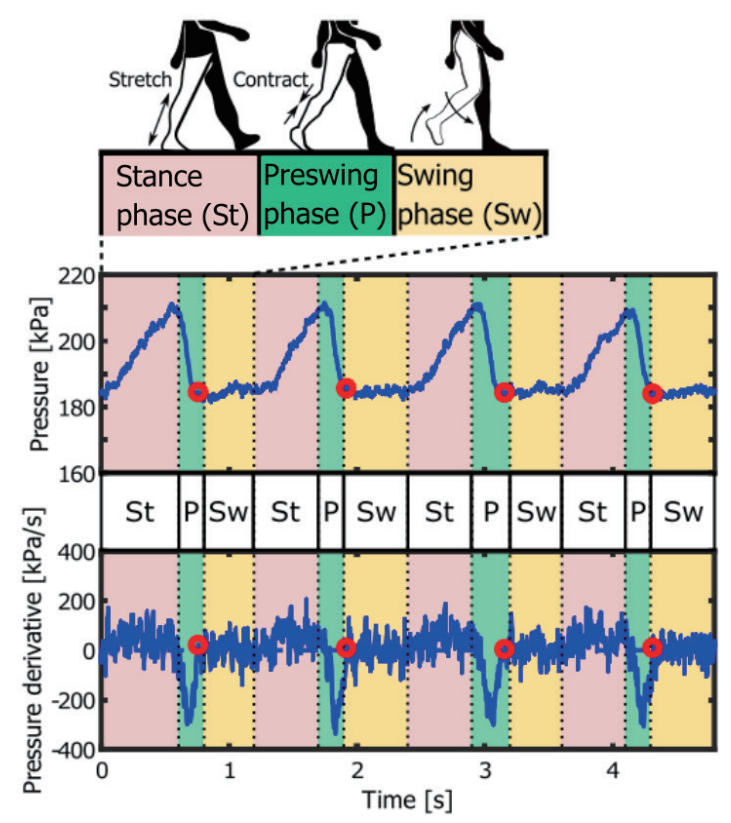

Fig. 4. (Color online) Experimental data sample of pressure and pressure derivative in RCB PARM. 
a low-pass filter whose cut-off frequency is $10 \mathrm{~Hz}$, which is assumed to be sufficiently high for measuring human gait motion. The pressure derivative data is calculated from the pressure data by using a numerical differentiation at a cut-off frequency of $10 \mathrm{~Hz}$. In Fig. 4, the red area is the stance phase (St), the green area is the preswing phase (P), and the yellow area is the swing phase (Sw).

The gait phase detection procedure is as follows:

1. The time step $k_{1}$ is detected as the time when the pressure derivative of the CB PARM $\dot{P}_{c}(\mathrm{kPa} / \mathrm{s})$ crosses its threshold $\dot{P}_{t h}(\mathrm{kPa} / \mathrm{s})$.

2. The measured pressure of the CB PARM $P_{c 1}(\mathrm{kPa})$ at the time step $k_{1}$ is recorded, and the previously detected $k_{1}$ and $P_{c 1}$ are set as $k_{2}$ and $P_{c 2}(\mathrm{kPa})$, respectively.

3. The difference between $P_{c 1}$ and $P_{c 2}$ is calculated as $\Delta P_{c}=P_{c 1}-P_{c 2}(\mathrm{kPa})$. When $\Delta P_{c}$ is less than the threshold $\Delta P_{t h}, k_{1}$ is set as the assist timing $k_{a}$.

4. Return to step 1 and repeat these steps.

In Fig. 4, the red marks show the assist timing $k_{a}$ detected by the above procedure, and it can be seen that the end timing of the preswing phase is detected in each gait cycle. The thresholds $\dot{P}_{t h}$ and $\Delta P_{t h}$ are set to detect the preswing phase timing. As shown in Fig. 4, the pressure derivative becomes negative and changes to positive in the preswing phase. Therefore, we set the pressure derivative threshold $\dot{P}_{t h}$ as $0 \mathrm{kPa} / \mathrm{s}$. In addition, there is a large pressure decrease in the preswing phase, and the pressure difference threshold $\Delta P_{t h}$ is set to detect the time, that the pressure becomes minimum in the preswing phase. We set the pressure difference threshold $\Delta P_{t h}$ as $-5 \mathrm{kPa}$ in Fig. 4. $\Delta P_{t h}$ should be configured in each trainee appropriately. The large $\Delta P_{t h}$ will be effective to avoid wrong detection; however, $\Delta P_{t h}$ should be lower than the pressure change amount in the preswing phase. The gait phase detection procedure is carried out for each leg independently.

\subsection{PARM control for load increase training}

The timing of the preswing phase is detected by using the method described in Sect. 3.1, and gait cycle time can be calculated from the detected timing. ${ }^{12)}$ In this study, we consider the torque trajectories of the hip, knee, and ankle joints during walking ${ }^{(14)}$ and drive the PARMs to increase the leg load. Each joint torque trajectory is shown in Fig. 5. In Fig. 5, the upper left figure is the trajectory of the hip joint, the upper center figure is that of the knee joint, and the upper right figure is that of the ankle joint. St is the stance phase, $\mathrm{P}$ is the preswing phase, and $\mathrm{Sw}$ is the swing phase. The sign of each joint torque is shown in the lower center figure of Fig. 5. The hip and knee joint torque of extension motion is defined as positive, and the ankle joint torque of plantarflexion motion is also positive. The torque in the opposite direction is negative. The maximum torque of the hip and ankle joints is larger than that of the knee joint and bears the principal driving torque for the gait motion.

Therefore, we consider giving the leg load by designing the target torque whose direction is opposite to the torque trajectories of the hip and ankle joints. The pressurized timing of each joint is shown in Fig. 5 (lower left and lower right). By driving the PARMs at the timing 

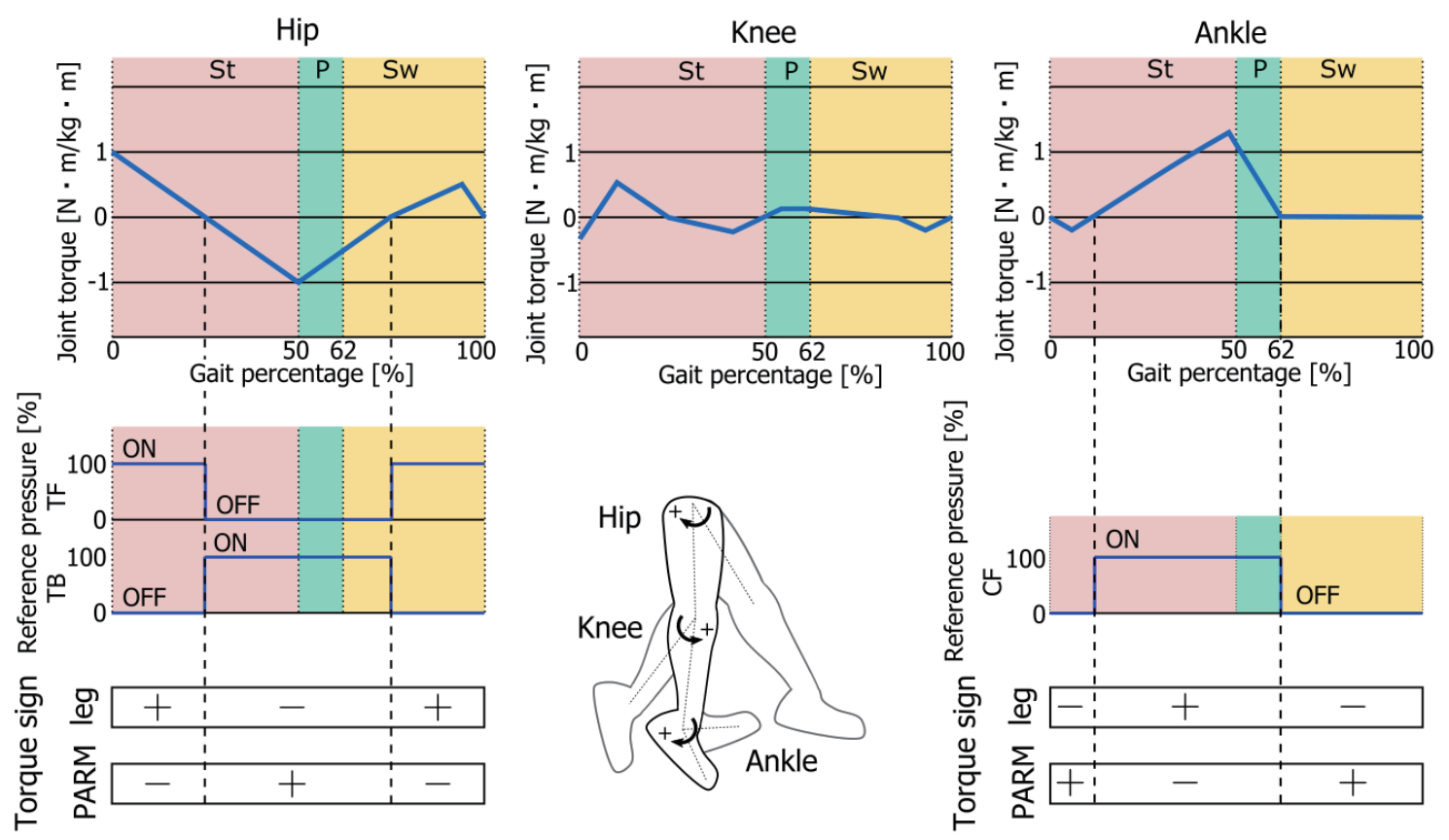

Fig. 5. (Color online) Target pressure trajectory for leg load increase considering the sign of the joint torque trajectory.

shown in Fig. 5, the load is effectively applied to the leg joints during walking. The TF PARM generates the torque that contributes to hip flexion (negative direction) and knee extension (positive direction) during its contraction. The TF PARM is pressurized between 0 to $25 \%$ and 75 to $100 \%$ of the gait cycle and gives a load on the hip joint. The TB PARM generates torque in the opposite direction to the TF PARM; therefore, it is driven at the opposite time of the TF PARM and pressurized between 25 to $75 \%$ of the gait cycle. When the CF PARM contracts, the contraction force dorsiflexes the ankle joint (negative direction). Therefore, the CF PARM is pressurized between 12 and $62 \%$ of the gait cycle to give the load on the ankle joint. The CB PARM is a pressurized closed chamber similar to a balloon and contracts throughout the gait cycle. The CB PARM generates torque that contributes to knee flexion (negative direction) and ankle plantarflexion (positive direction) during its contraction. Considering the sign of the ankle torque trajectory in Fig. 5 (lower right), the contraction force of the CB PARM assists in the plantarflexion of the ankle in the stance and preswing phases. On the other hand, it also becomes a load that prevents the ankle dorsiflexion in the swing phase.

The PARMs other than CF PARM contribute to the knee joint torque, and the load on the knee joint is determined by the drive timing of TF, TB, and CB PARMs. During 0 to $25 \%$ and 75 to $100 \%$ of the gait cycle, the extension torque due to the TF PARM and the flexion torque due to the CB PARM act on the knee joint. Since these are opposite torques and cancel each other, the load on the knee joint will become small. During 25 to $75 \%$ of the gait cycle, the flexion torque due to both the TB and CB PARMs acts on the knee joint, and a large load will be applied during knee extension. 
Note that the proposed training method is essentially based on an assumption that the trainee performs normal walking as described in the conventional study. ${ }^{(14)}$ We describe this reason as follows. If the trainer does not give any instructions about the gait to the trainee, wearing the gait-assistive suit and applying the external force may change both the trainee's gait kinematics and leg muscle activity. However, in the proposed training method, if the gait kinematics change is allowed, the trainee will be able to walk without any external force since the reference pressure is predefined as shown in Fig. 5 and dose not change along with the gait kinematics change. We considered that it will be difficult to apply an effective load to the leg muscle activity. Therefore, in this study, we focused on enhancing leg muscle activity as the main target of our proposed training method. To achieve the target, an experiment is carried out under intentional constraints so that the trainee's gait kinematics will not change in both cases with and without the external force. The constraints are described concretely in Sect. 4.1.

\section{Experiment}

\subsection{Experimental method}

To validate the effectiveness of the proposed load increase training using the gait-assistive suit, we carried out an evaluation experiment. In this experiment, two trainees wore the gaitassistive suit and we measured their gait. Trainee 1 is a healthy male in his $30 \mathrm{~s}$ with a height of $171 \mathrm{~cm}$ and a weight of $75 \mathrm{~kg}$. Trainee 2 is a healthy male in his $20 \mathrm{~s}$ with a height of $176 \mathrm{~cm}$ and a weight of $73 \mathrm{~kg}$. The research protocol was reviewed and approved by the ethical review board of Tokyo Medical and Dental University. Written informed consent was obtained from the trainees, including consent to participate and to publish the findings.

In the experiment, the trainee walks on the treadmill, and the EMG signal of the leg muscles is measured and used for the load evaluation. Note that the measured EMG signal is only used for the load evaluation, and it is not used for the suit control. The EMG signal is measured by using the EMG sensor (Delsys Inc., Bagnoli desktop EMG systems). The measurement frequency of the EMG signal was set as $500 \mathrm{~Hz}$. The trainee's gait is assumed to be a symmetric motion. The four EMG sensors are placed on the target muscles of the right leg, which include the vastus lateralis (RTF), semitendinosus (RTB), gastrocnemius (RCB), and tibialis anterior (RCF). The root-mean-square (RMS) value $J_{r}(\mathrm{~V})$ of the measured EMG signal in each gait cycle is calculated as

$$
J_{r}=\sqrt{\frac{1}{T_{c}} \int_{0}^{T_{c}} E M G_{(t)}^{2} d t},
$$

where $T_{c}(\mathrm{~s})$ is one gait cycle time, and $E M G(\mathrm{~V})$ is the measured EMG signal at time $t(\mathrm{~s})$. The noise of the EMG signal caused by cable swinging is removed by using a high-pass filter whose cutoff frequency is $25 \mathrm{~Hz}$. The RMS value in the maximum voluntary contraction (MVC) state is also measured and its value is used to normalize the RMS value in each channel. The normalized RMS value is defined as $\% M V C(\%)$, and it is calculated in each channel as 


$$
\% M V C=\frac{J_{r}}{J_{r}^{M V C}} \times 100
$$

where $J_{r}^{M V C}(\mathrm{~V})$ is the RMS value in the MVC state. The sensor positions and the clinical test method of measuring the MVC state are determined on the basis of the SENIAM project. ${ }^{(15)}$

As described in Sect. 3.2, the experiment was carried out under intentional constraints so that the trainee's gait kinematics would not change owing to the external force. Concretely, we set the following two constraints: (i) a metronome was used to maintain the walking rate, and (ii) the trainees were instructed as follows: "Even when you feel a load when walking due to the external force, please walk in your normal form." Since we set these constraints, we assume that the trainee walked with the same gait and form regardless of the external force. Therefore, kinematic analysis of the cases with and without the external force was not considered, and only leg muscle activity was analyzed in the experiment. The metronome was set at a tempo the trainee felt comfortable. In this study, the walking rates were set at 90 beats per minute (BPM) for trainee 1 and 110 BPM for trainee 2. The trainee walked on the treadmill (20 cycles of 40 steps each) at a speed of $4.0 \mathrm{~km} / \mathrm{h}$ in each trial.

We set the parameters as follows. The pressure in the CB PARM used for phase detection was set at $400 \mathrm{kPa}$, with the trainee standing upright on a flat floor without the CB PARM slacking. The target pressures in the PARM controlled by the servo valve were set at $0 \mathrm{kPa}$ (OFF mode in Fig. 5) and $400 \mathrm{kPa}$ (ON mode in Fig. 5). Concerning the pressure feedback controller, the proportional gain $K_{p}$ was set at $3.0 \times 10^{-2} \mathrm{~V} / \mathrm{kPa}$, and the integral gain $K_{i}$ was set at $7.0 \times 10^{-2} \mathrm{~V} / \mathrm{kPa}$ s. For gait phase detection, the pressure derivative threshold $\dot{P}_{t h}$ was set at 0 $\mathrm{kPa} / \mathrm{s}$, and the pressure difference threshold $\Delta P_{\text {th }}$ was set at $-15 \mathrm{kPa}$.

We measured under the following two conditions: (a) the trainee walked without wearing the suit, (b) the trainee wearing the suit walked against the external load. The experimental procedure was as follows:

1. Attach the EMG sensors onto the trainee. Perform the EMG measurement test while adjusting the sensor position to obtain a clear signal.

2. Rest for 5 min.

3. Measure the RMS value in the MVC state $J_{r}^{M V C}$.

4. Rest for $5 \mathrm{~min}$.

5. Measure the EMG of the gait without the suit.

6. Wear the suit.

7. Practice walking against the external load.

8. Rest for $10 \mathrm{~min}$.

9. Measure the EMG of the gait with the external load applied from the suit.

10. End

\subsection{Experimental result}

The trainee could walk at a constant pace in synchrony with the tempo of the metronome in both cases with and without external force. Figure 6 shows an example of the gait sequence 


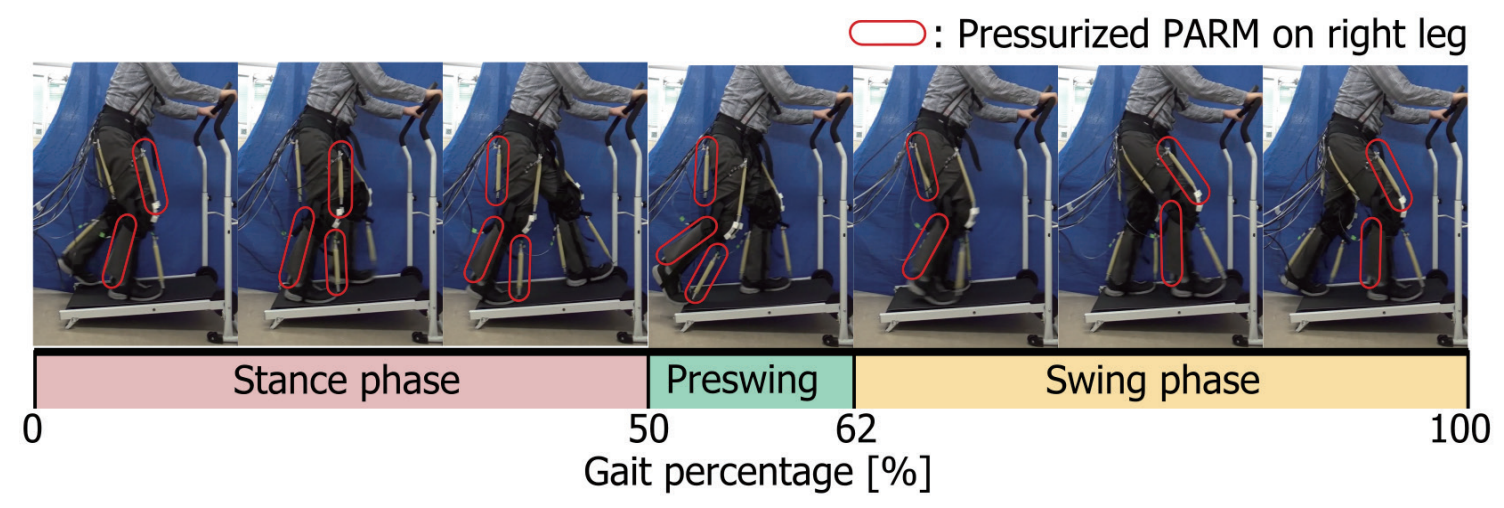

Fig. 6. (Color online) Gait sequence with the external load applied from the suit.

with the external load applied from the suit. The horizontal axis shows the gait percentage of the right leg. The red oblong marks indicate the pressurized PARM on the right leg. The TF and TB PARMs are pressurized alternately as described in Sect. 3.2. These PARMs are actuated to prevent thigh motion. The CF PARMs are pressurized from the middle stance phase to the end of the preswing phase to prevent the kicking motion of the toes. The CB PARMs are closed pressurized chambers, and their contraction force is transmitted to the trainee's leg through the whole gait cycle.

The results of EMG measurement are shown in Fig. 7. The vertical axis is the \%MVC value, and the horizontal axis is the measured position. The black bars are the mean \%MVC values of the gait without the suit, and the white bars are those of the gait with the external load applied from the suit. The dotted line bars are the standard deviations. The \%MVC values were calculated from the data of each gait cycle, and their mean value and the standard deviation were obtained from the 20-cycle data. We tested the significance of the difference in the measured data using Welch's t-test. The symbol * indicates that the values are statistically significantly different at the significance level of $1 \%$ determined using the two-sided test.

In addition, Fig. 8 shows the experimental data samples of the pressure and EMG signal of trainee 1. In the upper figures, the solid and dashed lines indicate the measured pressure and the reference pressure, respectively. In the lower figures, the blue and green lines are the EMG signals with and without the load of the suit, respectively. St is the stance phase, $\mathrm{P}$ is the preswing phase, and $\mathrm{Sw}$ is the swing phase.

\subsection{Discussion}

We discuss the experimental results shown in Fig. 7. In the case of these trainees, we confirmed that all the eight channels measured showed a significant difference. Although the RCB channel of trainee 1 and the RCF channel of trainee 2 have a small difference between the black and white bars relative to the other channels, their standard deviations were small.

Since the \%MVC values of all the RTF and RTB channels increased significantly, we confirmed that the proposed training method increased the load on the hip. In Fig. 8(a), 


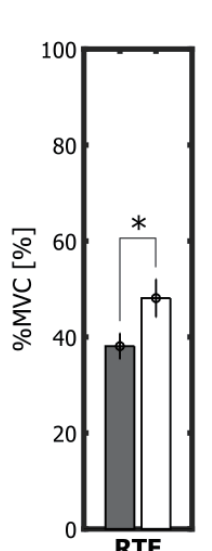

RTF
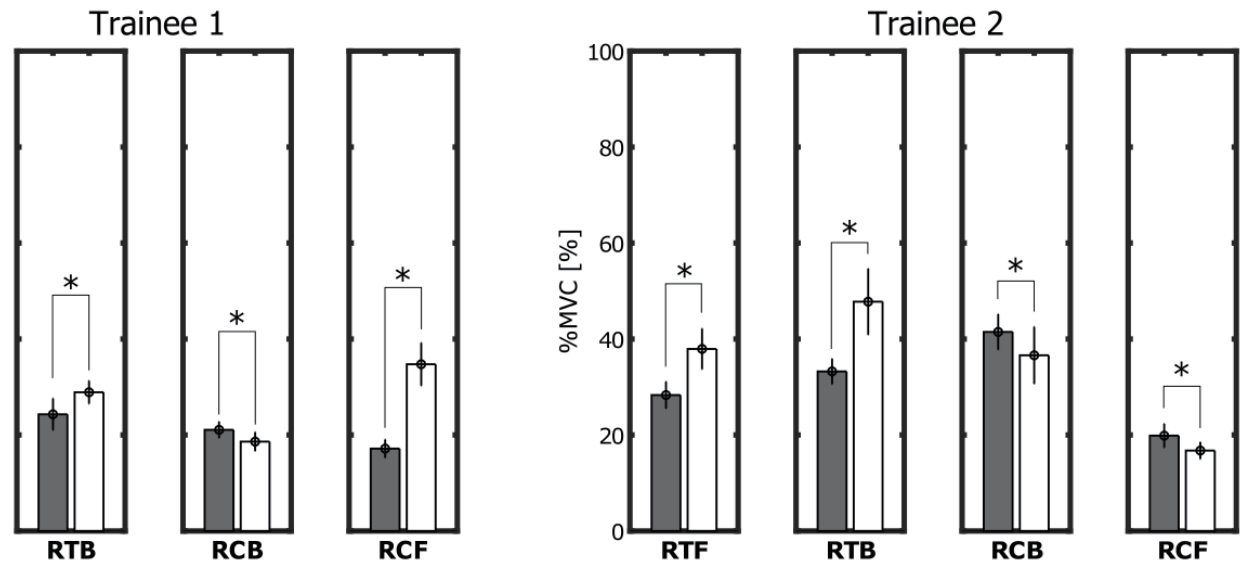

Fig. 7. Experimental results of the EMG measurement. The black bold bars are the mean \%MVC values of the gait without the suit, and the white bold bars are those of the gait with the external load applied from the suit. The dotted white bars are the standard deviations. The symbol * indicates that the values are statistically significantly different at the significance level of $1 \%$ using the two-sided test.

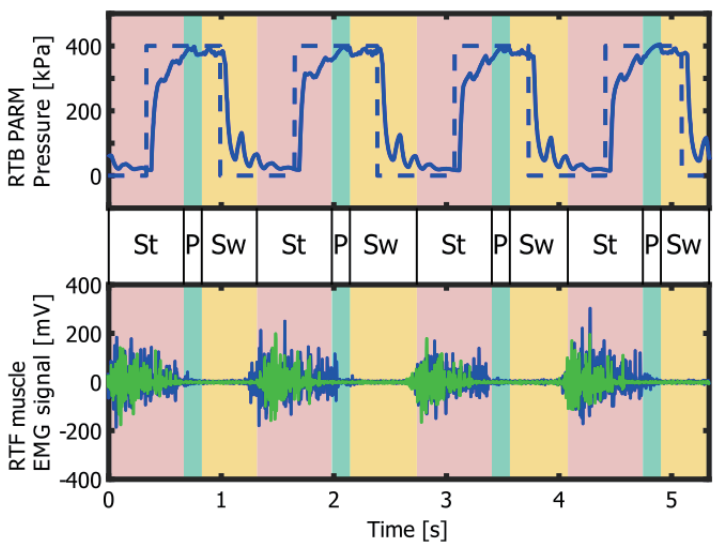

(a)

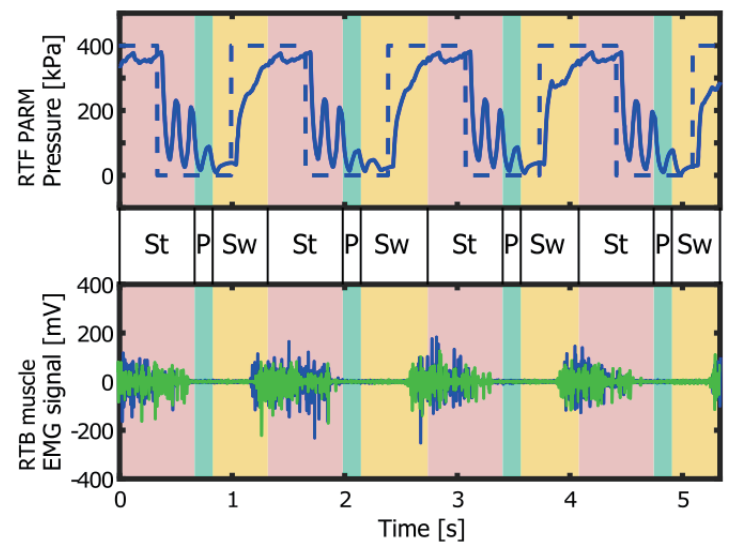

(b)

Fig. 8. (Color online) Experimental data samples of the pressure and EMG signal of trainee 1. In the upper figures, the solid and dashed lines are the measured pressure and reference pressure. In the lower figures, the blue and green lines are the EMG signals with and without the load of the suit, respectively.

when the RTB PARM was pressurized, the EMG signal of the RTF muscle with the load was activated longer than that without the load. In Fig. 8(b), when the RTF PARM was pressurized, the EMG signal of the RTB muscle with the load became slightly larger than that without the load.

On the other hand, the \%MVC value of RCB decreased, which means that the load on the ankle plantarflexion decreased. The plantarflexion torque was always applied to the ankle joint from the RCB PARM, which is a closed pressurized chamber. During the pressurization of the RCF PARM, the torques of the RCB and RCF PARM cancel each other, and the load on the ankle joint is temporarily reduced. The \%MVC value of the RCB channel was not increased 
since the load in the dorsiflexion direction was canceled as mentioned above. Therefore, we considered that the load in the plantarflexion direction is mainly applied to the ankle joint.

In the case of trainee 1 , the $\% \mathrm{MVC}$ value of the RCF channel that measures the activity of the tibialis anterior increased to resist the load on the plantarflexion. On the other hand, in the case of trainee 2, the \%MVC value of the RCF channel was decreased when the trainee wore the suit. We considered that the reason is that trainee 2 did not try to resist the load on the plantarflexion, and his gait changed, whivch was similar to walking on tiptoe to overcome the load. This type of gait makes the RCB PARM short and its contraction force small. This result suggests the following two points: (a) the given intentional constraints (i) and (ii) ultimately depend on the trainee's effort, and (b) the pressure in the PARM should be set appropriately to the extent that the trainee can walk in the normal form.

To increase the \%MVC values of both the RCB and RCF channels, it will be effective that the timing detection function and the load adding function are assigned to different PARMs. The PARM for the timing detection should have a small diameter and a small output force so that a large load is not applied to the ankle even when the pressure is always applied. Moreover, the PARM for applying the plantarflexion torque on the ankle should have a large diameter. Although this configuration increases the number of PARMs, it will be possible to add the load in the plantarflexion and dorsiflexion directions to the ankle joint at individual timings.

\section{Conclusion}

In this paper, we proposed a leg load increase training method using a gait-assistive suit. As the method for increasing the gait load, we focused on the torque trajectory of the leg joint during walking, and the gait-assistive suit generates the counter-torque on the leg joint using the contraction force of the PARM. To verify the proposed method, we carried out the EMG measurement experiment. In this experiment, the leg EMG of the trainee wearing the gaitassistive suit was measured, and its change caused by the applied load was observed. From the experimental results, we confirmed that the proposed training method increased the load on hip extension and flexion. On the other hand, we confirmed that the load on ankle plantarflexion was decreased, and the load on ankle dorsiflexion tended to increase in trainee 1 and tended to decrease in trainee 2. As a future work, we will improve the mechanism of the gait-assistive suit to increase also the ankle load. In addition, we consider that the load increase training method that allows the trainee's gait to change is an interesting and important research topic. We will improve the control algorithm of the gait-assistive suit in order to realize it.

\section{Acknowledgments}

This research was supported by Bridgestone Corporation and is based on the Cooperative Research Project of the Research Center for Biomedical Engineering. The authors would like to thank Shingo Oono, Ryo Sakurai, and Shintaro Yoshida of Bridgestone Corporation for their support. 


\section{References}

1 B. H. Goodpaster, S. W. Park, T. B. Harris, S. B. Kritchevsky, M. Nevitt, A. V. Schwartz, E. M. Simonsick, F. A. Tylavsky, M. Visser, and A. B. Newman: J. Gerontol. A 61 (2006) 1059. https://doi.org/10.1093/ gerona/61.10.1059

2 M. Iannuzzi-Sucich, K. M. Prestwood, and A. M. Kenny: J. Gerontol. A 57 (2002) M772. https://doi. org/10.1093/gerona/57.12.M772

3 A. C. Jozsi, W. W. Campbell, L. Joseph, S. L. Davey, and W. J. Evans: J. Gerontol. A 54 (1999) M591. https:// doi.org/10.1093/gerona/54.11.M591

4 W. R. Frontera, C. N. Meredith, K. P. O’Reilly, H. G. Knuttgen, and W. J. Evans: J. Appl. Physiol. 64 (1988) 1038. https://doi.org/10.1152/jappl.1988.64.3.1038

5 G. R. Hunter, C. J. Wetzstein, D. A. Fields, A. Brown, and M. M. Bamman: J. Appl. Physiol. 89 (2000) 977. https://doi.org/10.1152/jappl.2000.89.3.977

6 R. Orr, N. J. de Vos, N. A. Singh, D. A. Ross, T. M. Stavrinos, and M. A. Fiatarone-Singh: J. Gerontol. A 61 (2006) 78. https://doi.org/10.1093/gerona/61.1.78

7 A. Wernig and S. Müller: Paraplegia 30 (1992) 229. https://doi.org/10.1038/sc.1992.61

8 N. Takeshima, M. E. Rogers, E. Watanabe, W. F. Brechue, A. Okada, T. Yamada, M. M. Islam, and J. Hayano: Med. Sci. Sports Exerc. 33 (2002) 544. https://doi.org/10.1097/00005768-200203000-00024

9 Alter G Inc.: https://www.alterg.com (accessed August 2019).

10 Technogym: https://www.technogym.com/skillrun-performance-running.html (accessed September 2019).

11 T. H. Kim, and B. H. Hwang: J. Phys. Ther. Sci. 29 (2017) 2172. https://doi.org/10.1589/jpts.29.2172

12 T. Miyazaki, T. Tagami, D. Morisaki, R. Miyazaki, T.Kawase, T. Kanno, and K. Kawashima: Appl. Sci. 9 (2019) 2869. https://doi.org/10.3390/app9142869

13 T. Miyazaki, H. Suzuki, D. Morisaki, T. Kanno, R. Miyazaki, T. Kawase, Y. Kawakami, and K. Kawashima: Proc. 2019 IEEE/SICE 11th International Symposium on System Integration (IEEE/SICE, Paris, 2019) 237242. https://doi.org/10.1109/SII.2019.8700346

14 J. Perry and J. M. Burnfield: Gait Analysis: Normal and Pathological Function (Slack Inc., Thorefare, NJ, 2010) ISBN 978-1-55642-766-4

15 The SENIAM Project: http://www.seniam.org (accessed August 2019).

\section{About the Authors}

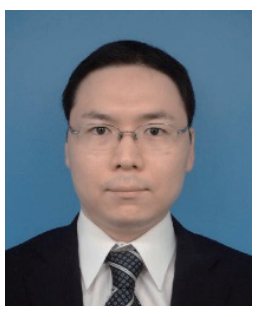

Tetsuro Miyazaki received his doctoral degree in engineering from the Department of Mechanical Sciences and Engineering of Tokyo Institute of Technology in 2014. From 2014 to 2017, he worked as a research assistant (2014 to 2015) and an assistant professor (2015 to 2017) at Yokohama National University. Since April 2017, he has been an assistant professor at the Institute of Biomaterials and Bioengineering of Tokyo Medical and Dental University. His research interests are mechanical engineering, control engineering, power assistive device, and medical welfare robotics. (tmiyazaki.bmc@tmd.ac.jp)

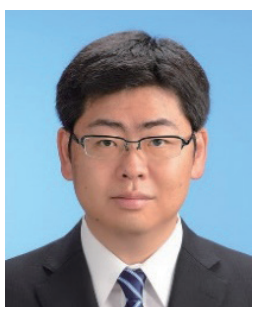

Toshihiro Kawase received his B.S., M.S., and Ph.D. degrees from Tokyo Institute of Technology, Tokyo, Japan in 2007, 2009, and 2012, respectively. He was a research fellow at the Research Institute of National Rehabilitation Center for Persons with Disabilities from 2012 to 2015, and worked as a postdoctoral fellow and a specially appointed assistant professor at Tokyo Institute of Technology from 2015 to 2017 . He is currently an assistant professor at Tokyo Medical and Dental University and Tokyo Institute of Technology. His research interests include rehabilitation robotics, medical robots, and biological signal processing. (kawase.bmc@tmd.ac.jp) 


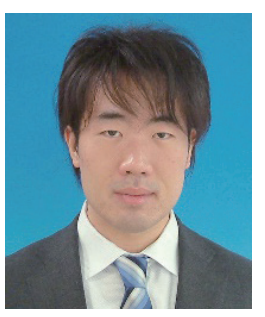

Takahiro Kanno received his doctoral degree in engineering from the Department of Mechanical Engineering and Science of Kyoto University in 2013. In 2013, he worked as a postdoctoral researcher in the Precision and Intelligence Laboratory, Tokyo Institute of Technology. From 2013 to 2019, he worked as an assistant professor since June 2019, he has been an associate professorat Tokyo Medical and Dental University. His research interests are medical robotics, control engineering, and teleoperation.

(kanno.bmc@tmd.ac.jp)

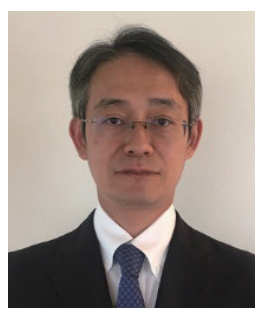

Kenji Kawashima received his doctoral degree in engineering from the Department of Control Engineering of Tokyo Institute of Technology in 1997. From 1997 to 2000, he worked as a research assistant at Tokyo Metropolitan College of Technology. He then worked as an associate professor in the Precision and Intelligence Laboratory of Tokyo Institute of Technology. Since April 2013, he has been a professor at the Institute of Biomaterials and Bioengineering of Tokyo Medical and Dental University. His research interests are medical robotics, control engineering, and fluid measurement and control. (kkawa.bmc@tmd.ac.jp) 